\section{Arbeit orientiert sich an Problemen in der Praxis}

Für Dr. Juliane Gösling stellte sich die Weiche Richtung Berufspolitik gegen Ende ihrer Assistenzzeit, als die Entscheidung fallen musste: Selbständigkeit oder was? In dieser Zeit wurde sie auf den Studiengang „Public Health“ " an der Medizinischen Hochschule Hannover aufmerksam. Mit dieser Zusatzausbildung in der Tasche war sie dann bestens vorbereitet für ihren jetzigen Fulltimejob als Referentin im Bereich „Zahnärztliche Berufsausübung" bei der Bundeszahnärztekammer (BZ̈̈K). Zu ihren Arbeitsbereichen gehören unter anderem die Röntgenstelle, die Arzneimittelkommission und die Ausschüsse für Weiterbildung. Dabei beinhaltet ihr Arbeitsalltag viel Schreibtischarbeit, aber auch Veranstaltungen, Sitzungen und Gespräche. Aktuell ist sie z. B. an der Erstellung einer Broschüre für das Praxisteam mit dem Titel „Keine Angst vor HIV, HBV und HCV“ beteiligt, die die BZÄK in Zusammenarbeit mit der Deutschen Aidshilfe herausgeben wird. Die Broschüre liefert Informationen zu Übertragungswegen, Schutzmöglichkeiten und schafft damit die Grundlage für einen vorurteilsfreien Umgang mit Infizierten. „Die Arbeit orientiert sich also sehr an den in der Praxis auftauchenden Problemen", so Gösling.

\section{Politisch engagieren und trotzdem weiter bohren!}

Doch man muss sich nicht komplett von der praktischen Tätigkeit verabschieden, wenn man sich politisch engagieren will: Dr. Frank Hofmann ist Oralchirurg in eigener Praxis in Bitter-

\title{
„Ändern zu können, was einen geärgert hat - das ist ein tolles Gefühl!“
}

Interview mit Dr. Juliane Gösling, Referentin im Bereich "Zahnärztliche Berufsausübung" bei der Bundeszahnärztekammer

djz: Was genau waren Ihre Beweggründe für den Berufswechsel? Dr. Juliane Gösling: Auf jeden Fall keine Unzufriedenheit mit der praktischen Arbeit oder dem Praxisteam, damit hatte ich sehr viel Glück. Irgendwann musste aber die Entscheidung fallen, sich selbstständig zu machen oder einen anderen Weg einzuschlagen. Ich habe mich dafür entschieden, beruflich nochmal etwas anderes auszuprobieren und das habe ich bisher auch nicht bereut. Zugegebenermaßen hat die Möglichkeit nach Berlin zu gehen, auch zur Entscheidung beigetragen.

djz: Was schätzen Sie an Ihrem neuen/anderen Berufsalltag, was fehlt Ihnen?

Gösling: Mir persönlich macht die Arbeit an Projekten, wie beispielsweise die Überarbeitung der Broschüre „Schritte in das zahnärztliche Berufsleben“ viel Spaß. Die Broschüre hilft jungen Kolleginnen und Kollegen, sich nach dem Studium zu orientieren. Dabei kann ich auch meine persönlichen Erfahrungen im Hinblick, welche Informationen junge Kolleginnen und Kollegen brauchen, einbringen. Ein Vorteil gegenüber der Praxis ist, dass man die Zeit selbst einteilen kann, es gibt keine Patienten die warten, keine roten Anzeigen „der Patient wartet schon zehn Minuten“.

Was mir ein wenig fehlt ist der praktische Teil. Aber ich habe auch ein gutes Netzwerk aus ehemaligen Kommilitonen, die mich immer auf dem Laufenden halten. Ansonsten ist es ein tolles Gefühl, Dinge, die einen im Praxisalltag geärgert haben, mit angehen zu können. Natürlich ist der Handlungsspielraum beschränkt. Aber ich kann meinen Kollegen helfen, Abläufe, Strukturen etc. zu verbessern.

djz: Wie kann man gerade junge Kollegen zum Engagement in der Berufspolitik gewinnen?

Gösling: Über die eigene Praxis hinaus mitzugestalten und mitzuentscheiden, ist sehr interessant und auch verantwortungsvoll. Gerade in den Selbstverwaltungen, ob nun wie ich in einer Anstellung oder auch im Ehrenamt, brauchen wir eine breite und repräsentative Basis. Hier brauchen wir mehr junge Kolleginnen und Kollegen. Dafür mache ich immer wieder Werbung. Wichtig zu wissen: Ehrenamtlich bedeutet nicht umsonst. Auch hier gibt es einen Ausgleich. 